\title{
Wie fremdenfeindlich ist die Schweiz?
}

\section{Fünf Feldexperimente über prosoziales Verhalten und die Diskriminierung von Ausländern in der Stadt Zürich und in der Deutschschweiz}

\author{
Von Andreas Diekmann, Ben Jann und Matthias Näf
}

Zusammenfassung: Umfragen über Vorurteile führen oft zu verzerrten Ergebnissen, da die Befragten häufig „,sozial erwünschte“ Antworten geben. In unserer Studie über Vorurteile und Diskriminierung haben wir dagegen vier Feldexperimente in der Stadt Zürich durchgeführt, in denen jeweils von Angehörigen verschiedener Nationalitäten oder religiöser Gruppen eine Hilfeleistung erbeten wurde. Beispielsweise wurden Passanten von einer Person mit Kopftuch um eine Hilfeleistung gebeten; in der Kontrollbedingung dagegen ohne Kopftuch. In einem anderen Experiment wurden Personen auf Hochdeutsch angesprochen und in der Kontrollsituation im Schweizer Dialekt. Ein fünftes Experiment bezieht sich auf Bewerbungen bei Arbeitgebern in der Deutschschweiz. Es zeigte sich, dass im Alltagsverhalten keine signifikanten Unterschiede im Ausmaß der Hilfeleistung zwischen den Gruppen nachweisbar waren. Alle vier Experimente zu kleinen Hilfen im Alltag ergaben weder bezüglich Deutschen noch muslimischen Minderheiten Hinweise auf diskriminierendes Verhalten. Zu berücksichtigen ist allerdings, dass die Experimente in der Stadt Zürich durchgeführt wurden und das Ausmaß von Diskriminierung in anderen Regionen der Schweiz durchaus höher ausfallen könnte. Deutliche Hinweise gibt es dagegen für die Diskriminierung bestimmter ethnischer Gruppen auf dem Arbeitsmarkt, auch wenn Bewerber die gleiche Qualifikation wie Schweizer und die Schweizer Staatsbürgerschaft besitzen.

\section{Ziel der Untersuchung}

Der Anteil der Ausländerinnen und Ausländer an der Schweizer Wohnbevölkerung beträgt $23 \%$; in der erwerbstätigen Bevölkerung hat sogar mehr als jeder vierte Erwerbstätige keinen Schweizer Pass. Damit nimmt die Schweiz in Bezug auf den Ausländeranteil in Europa einen Spitzenplatz ein. Seit dem Freizügigkeitsabkommen mit der EU ist zudem ein erheblicher Zustrom gut qualifizierter Arbeitskräfte aus der Europäischen Union und insbesondere aus Deutschland zu beobachten. Dieser Zustrom massiert sich noch in der wirtschaftlich prosperierenden Agglomeration Zürich (Helbling 2011).

Infolge des Zustroms gab es in den Schweizer Medien eine heftige Debatte über Begrenzung der Einwanderung, Diskriminierung und das Freizügigkeitsabkommen mit der EU. Schweres Geschütz hatte die rechtskonservative Schweizerische Volkspartei (SVP) zunächst bei der Volksinitiative für die Ausschaffung krimineller Ausländer (,Ausschaffungsinitiative“; eingereicht 2008, angenommen 2010), bei einer Volksabstimmung über den Neubau von Minaretten („Minarettinitiative“; eingereicht 2008, angenommen 2009) und bei einer Initiative für demokratische Einbürgerungen („Einbürgerungsinitiative“; eingereicht 2005, abgelehnt 2008) aufgefahren. Bekannt wurde das Plakat, auf dem zu sehen war, wie ein schwarzes Schaf aus der Schweiz hinausgekickt wurde (Abbildung 1). Auf einem anderen Plakat greifen Menschen mit schwarzer Hautfarbe nach dem Schweizer Pass (,Einbürgerungsinitiative“). Auf dem Plakat zum Minarettverbot waren eine Burka-verhüllte Muslima und raketengleiche Minarette auf dem Schweizer Kreuz zu sehen. Die Kampagne zur Volksinitiative über kriminelle Ausländer hatte weltweite Empörung ausgelöst. Im Menschenrechtsrat in Genf hat der UNO-Sonderbeauftragte der Schweiz Rassismus vorgeworfen. Richteten sich diese Kampagnen gegen außereuropäische, insbesondere arabische und afrikanische Einwanderer, so nahm die SVP in einer späteren Kampagne die deutschen Zuwanderer ins Visier. Deutsche Ärzte und Professoren würden Inländern die Arbeitsplätze wegnehmen, lautet der Tenor. Berichte über eine tatsächliche oder angebliche Diskriminierung 
Deutscher häuften sich in den Medien. Der neueste Streich aus dem rechtskonservativen Lager war die „Masseneinwanderungsinitiative“, die vom Schweizer Souverän knapp angenommen wurde (eingereicht 2012, angenommen 9. Februar 2014) ${ }^{1}$.

Abbildung 1: Plakate der Schweizerischen Volkspartei

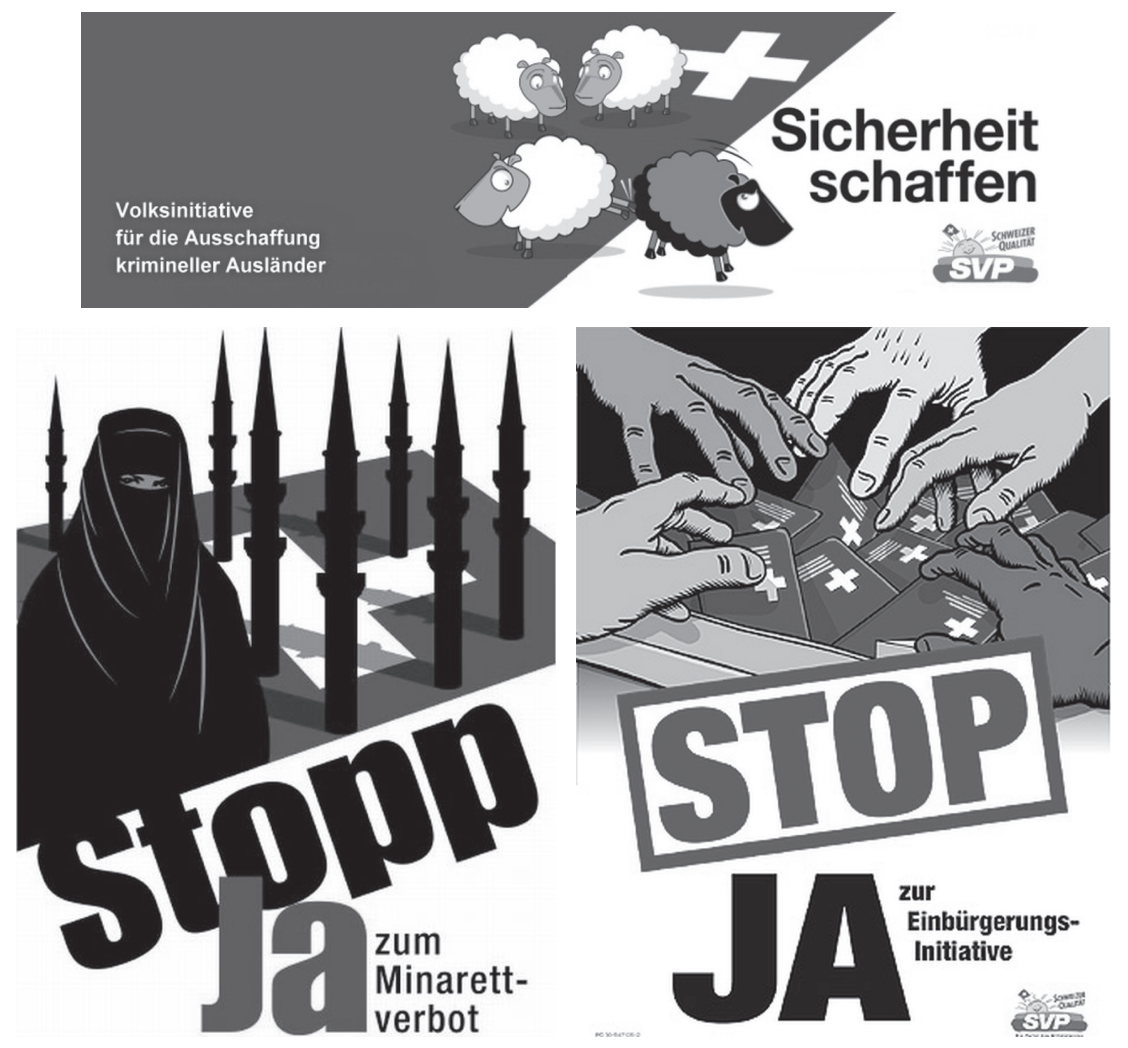

1 Die Initiative erhielt in der Stadt Zürich nur 33,4\% Ja-Stimmen; in der Innerschweiz dagegen rund $60 \%$. 


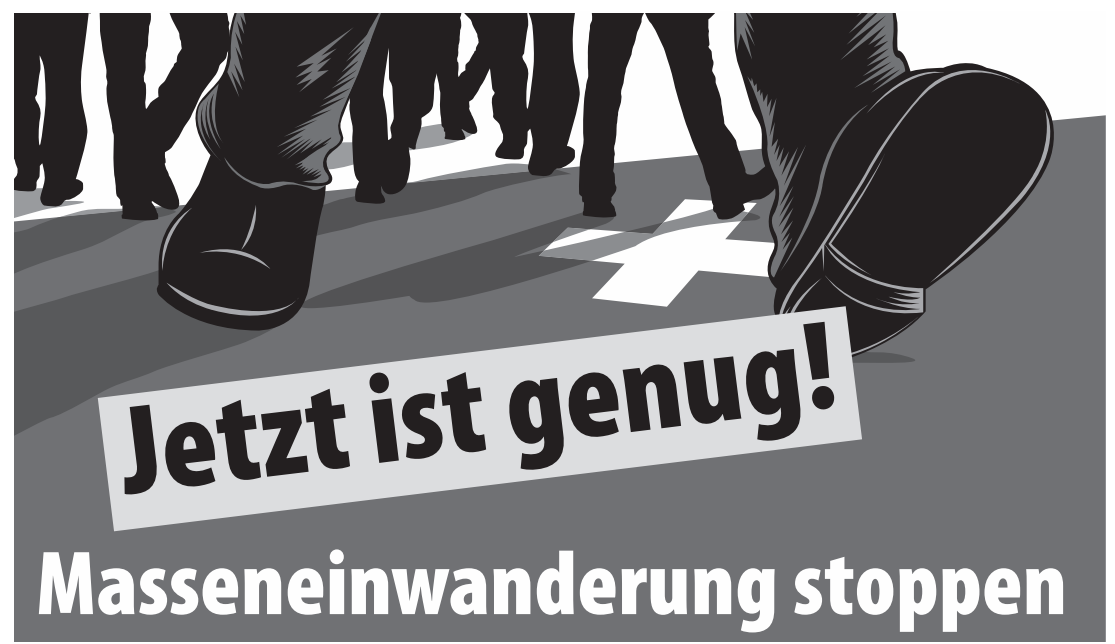

Wo immer Minderheiten zuströmen, die äußerlich durch Verhalten, Sprache, Kleidung oder andere Merkmale erkennbar sind und sich von den „Natives“ unterscheiden, kann es zu Spannungen kommen. Jüngst hat sogar der „Schwabenkrieg“ in Berlin Aufsehen erregt, wo Deutsche von Deutschen diskriminiert wurden, zugezogene Süddeutsche von „einheimischen" Berlinern. In der Sozialpsychologie haben die Experimente von Tajfel (Tajfel et al. 1971; Tajfel 1981) gezeigt, dass die Identifikation mit minimalen Gruppenzielen oder Symbolen ein Wir-Gefühl schafft und zur Abgrenzung von anderen Gruppen führt. In dem klassischen Experiment konnten Schüler zwischen einem Gemälde von Kandinski oder Klee wählen, ohne dass der Name des Malers genannt wurde. Je nach den geäußerten Vorlieben wurden Gruppen gebildet. Zu beobachten war, dass die Schüler bei der Aufteilung von Ressourcen Mitglieder der eigenen Gruppe bevorzugten und Mitglieder der anderen Gruppe diskriminierten (Tajfel et al. 1971). Weshalb tritt Diskriminierung so häufig auf? In einem Überblick zu Jahrzehnten psychologischer Vorurteilsforschung benennt Fiske (2000) mehrere Motive. Dazu zählen der Wunsch nach Zugehörigkeit zu einer sozialen Gruppe (,,belonging“), gemeinsames Verständnis von Werten und Verhaltensweisen der Gruppe (,,understanding“), Kontrolle von Umwelt und Interaktionen (,controlling“), das Selbstwertgefühl durch Zugehörigkeit (,self-enhancing“) und Vertrauen in die Zugehörigen der eigenen Gruppe (,trusting“). Allerdings wurden auch immer wieder Möglichkeiten zum Abbau von Vorurteilen diskutiert. Prominent ist die Kontakthypothese von Gordon Allport, die bereits vor sechs Jahrzehnten formuliert wurde: „It has sometimes been held that merely by assembling people without regard for race, color, religion, or national origin, we can thereby destroy stereotypes and develop friendly attitudes" (Allport 1954, zitiert nach Hewstone 2003). Tatsächlich zeigen umfangreiche Metaanalysen von Pettigrew und Tropp (2008), dass Kontakte zwischen Personen aus verschiedenen kulturellen und sozialen Gruppen dazu verhelfen, Vorurteile abzubauen. Selbstverständlich erweist sich die Kontakthypothese nur dann als wirksam, wenn soziale Interaktionen zwischen unterschiedlichen sozialen und kulturellen Gruppen auch tatsächlich häufig zustande kommen, wenn also Zuwanderer und Einheimische möglichst oft miteinander interagieren. Eine oft zu beobachtende starke Segregation der Kulturen wird dem Abbau von Vorurteilen und Diskriminierung dagegen kaum förderlich sein.

Nach Tajfels Studien können Vorurteile durch identitätsstiftende Symbole selbst im Labor erzeugt werden. Dies gilt umso mehr, wenn wichtige Ressourcen ins Spiel kommen oder zu- 
mindest Verteilungsprobleme knapper Ressourcen subjektiv wahrgenommen werden. Beim „Schwabenkrieg“ in Berlin geht es um die angebliche oder tatsächliche Gentrifizierung von Wohnraum, in Zürich werden das Arbeitsplatzargument oder steigende Mieten und Immobilienpreise geltend gemacht. Ob diese Vorwürfe zutreffen oder nicht, spielt keine Rolle, denn allein die subjektive Wahrnehmung kann die Diskriminierung von Minderheiten auslösen. Dabei werden die Themen oft von den Medien übernommen, die gleichzeitig als Echo und Verstärker fungieren können ${ }^{2}$. Als ein Beispiel von vielen sei eine Reportage im Magazin des Tagesanzeigers genannt. Hier wird mit anekdotischen Berichten vom Hörensagen ein Bild produziert, dass weniger qualifizierte Deutsche Einheimischen hoch dotierte Stellen wegnähmen. „Seit alle zwölf Monate 70.000 bis 80.000 Leute in die Schweiz ziehen, viele gut qualifiziert, werden die Mittelschichten zunehmend nervös", lautet ein Fazit des Autors (Beglinger 2013). Aber auch Deutsche fühlen sich diskriminiert, wie Leserbriefe demonstrieren. ${ }^{3}$ Nur sind das, ebenso wie die anekdotische Berichterstattung in Schweizer Zeitungen, Einzelfälle, die keinerlei generalisierende Aussagekraft haben.

Im Einklang mit der These der Verdrängungsängste kann Helbling (2011) anhand von Umfragedaten einer Stichprobe der Züricher Bevölkerung zeigen, dass die Einstellung gegenüber Deutschen negativer ausfällt, wenn die befragten Personen im Beruf aufstiegsorientiert sind. Außerdem ist nach seiner Studie bemerkenswert, dass - anders als bei Vorurteilen gegenüber Immigranten aus Ex-Jugoslawien - höhere Bildung nicht dazu beiträgt, den Grad einer negativen Einstellung gegenüber Deutschen abzuschwächen. Absolut gesehen sind es aber insbesondere Zuwanderer aus Ex-Jugoslawien, die unter Vorurteilen leiden müssen. Dies zeigt auch eine ältere Befragung in Zürich, die Mitte der 90er Jahre durchgeführt wurde (Stolz 2000, zitiert nach Helbling 2011). Ablehnende Einstellungen gegenüber Deutschen werden zwar häufiger geäußert als gegenüber Zuwanderern aus Italien, Spanien, Portugal oder Frankreich; wesentlich weiter verbreitet sind aber Vorurteile gegenüber Zuwanderern aus der Türkei und Ex-Jugoslawien. Negative Einstellungen zu Deutschen äußerten etwa $10 \%$ der Befragten, gegenüber jugoslawischen Immigranten waren es etwa 50\%.

Weshalb sind die Deutschen in der Deutschschweiz weniger beliebt als Italiener, Spanier, Portugiesen und Franzosen (wobei die Differenzen hier aber im einstelligen Prozentbereich liegen)? Helbling (2011) vermutet im Anschluss an die Theorie von Jetten, Spears und Manstead (1998), dass sowohl geringe als auch große Unähnlichkeit einer anderen sozialen Gruppe zu negativen Einstellungen führen kann; der Zusammenhang zwischen einer positiven Einstellung und der Ähnlichkeit einer Fremdgruppe also kurvilinear ist (Jetten et al.

2 Einer der Autoren (AD) wurde von einer Tageszeitung gebeten, zu den Diskussionen Stellung zu nehmen und eigene Diskriminierungserfahrungen zu berichten. Nach dem Hinweis, dass keine solchen eigenen Erfahrungen vorlägen, hatte die Zeitung kein Interesse mehr an der Stellungnahme.

3 Über das „Täschligate“, der von Oprah Winfrey in Zürich erfahrenen tatsächlichen oder vermeintlichen Diskriminierung, berichtete u.a. Spiegel-Online. Die prominente Afro-amerikanische TV-Moderatorin fühlte sich beim Einkauf einer Luxus-Handtasche diskriminiert. Von den zahlreichen Zuschriften an Spiegel-Online befassen sich nur sehr wenige mit der Diskriminierung von Deutschen. Die folgenden beiden Leserbriefe berichten Erfahrungen mit Diskriminierung: „Ich lebe als Ausländer in der Schweiz und ich muss ehrlich sagen, dass ich hier oft auf Ausländerfeindlichkeit treffe. Rassismus überhaupt nicht, aber immer wieder merke ich, dass man als Ausländer pauschal schlechter behandelt wird. Tut mir Leid liebe Schweizer, aber das empfinde nicht nur ich so, vielleicht solltet ihr euch wirklich Gedanken machen ...“ (thomas310). Und janne2109 schreibt: „Es war mit Sicherheit kein Missverständnis, und ja - man kann den Schweizern einen leichten Rassismus vorwerfen. Der richtet sich aber nicht nur gegen Farbige, sondern an alle Ausländer, übrigens auch Deutsche. Deutsche Mitarbeiterinnen (es gibt ja nur wenige) bei Aldi werden z.B. von den Schweizerinnen ebenfalls nicht freundlich in der Ansprache behandelt, ich habe es selbst erlebt" (Spiegel Online 11.8.2013). 
1998). Sprache und Kultur sind ähnlich, aber es gibt doch Unterschiede, wobei die Sprache, also Schweizer Dialekt versus Hochdeutsch eine wichtige Rolle spielt (Helbling 2011).

Vorurteile gegenüber Minderheiten werden in der Sozialforschung zumeist durch Umfragen erhoben. Diese Erhebungen kann man zwar verfeinern, indem spezielle Techniken der Anonymisierung bei heiklen Fragen eingesetzt werden (Krumpal 2012). Allerdings ist bekannt, dass oft ausgeprägte Diskrepanzen zwischen Einstellungen und Verhalten bestehen. Uns geht es daher um das tatsächliche Verhalten in Alltagssituationen. So genannte nichtreaktive Erhebungsverfahren und Feldexperimente (Webb et al. 1966; Bungard / Lück 1974; Diekmann 2007) können über das tatsächliche Ausmaß der Diskriminierung in Alltagssituationen Auskunft geben.

Wir fragen also, ob sich Diskriminierung in Alltagssituationen gegenüber Deutschen und anderen Ausländern zeigt und wenn ja, in welchem Ausmaß diese besteht. So könnte es sein, dass sich nur eine kleine Minderheit der alteingesessenen Bevölkerung diskriminierend verhält, die große Mehrheit dagegen Ausländer egalitär behandelt. Weiterhin könnte es sein, dass der Medienhype über die Diskriminierung von Deutschen nicht mehr als ein ,Sturm im Wasserglas' war, quasi ein sozial konstruiertes Bild von Diskriminierung, für das bei näherer Betrachtung keine wirkliche Entsprechung im tatsächlichen Verhalten der Bevölkerung aufzufinden ist.

Wir untersuchen mögliches diskriminierendes Verhalten mit fünf Feldexperimenten, die von studentischen Arbeitsgruppen eines Seminars oder als Semesterarbeit durchgeführt wurden. ${ }^{4}$ Alle Experimente wurden im Frühjahr 2010 durchgeführt; vier davon in Zürich, ein weiteres bei Firmen in der Deutschschweiz. Die ersten beiden Experimente beziehen sich auf eine mögliche Diskriminierung von Deutschen, die weiteren drei Experimente auf muslimische Migranten und Ausländer aus dem ehemaligen Jugoslawien. Die Kontrollgruppe bilden jeweils Inländer. Wir erläutern im Folgenden die Feldexperimente und Ergebnisse und diskutieren anschließend die Befunde.

\section{Fünf Feldexperimente}

\section{Experiment 1: „Häsch mer zwöi Stutz?"5}

Eine Bitte um eine kleine Hilfeleistung wird einmal auf Schweizerdeutsch, einmal auf Hochdeutsch vorgebracht. Werden Zürcher eher auf eine Bitte zu einer kleinen Hilfeleistung reagieren, wenn diese auf Schweizerdeutsch vorgebracht wird? Die Hypothese lautet, dass Hochdeutsch unbewusste Abwehrreflexe auslöst und sich dadurch die Wahrscheinlichkeit verringert, dass der Bitte entsprochen wird.

Das Experiment fand auf öffentlicher Straße im Zürcher Stadtzentrum statt. Willkürlich ausgewählte Passanten wurden um einen fehlenden Geldbetrag zum Kauf eines Tramfahrscheins gebeten. Der Betrag wurde nach einem Pretest auf zwei CHF festgelegt. Die Bitte wurde jeweils Wort für Wort gleich formuliert und entweder auf Hochdeutsch oder auf Schweizerdeutsch vorgetragen. Der Standardsatz lautete: „Entschuldigen Sie, darf ich Sie um einen kleinen Gefallen bitten? Es ist mir wirklich peinlich, aber ich brauche noch zwei Franken für einen Tramfahrschein nach Wollishofen.“

4 Die Daten und eine Dokumentation der fünf Feldexperimente (Jann 2014) sowie die studentischen Arbeiten stehen unter http://www.socio.ethz.ch/research/datafiles zur Verfügung. An gleicher Stelle findet sich ein Anhang mit einigen Zusatzanalysen.

5 Die Arbeitsgruppe bestand aus Melanie Bieli, Felicitas Hoppeler, Olivia van der Reijden, Luca Weber und Christopher Zimdars (siehe Zimdars et al. 2010). 
Würden verschiedene Personen die Bitte vorbringen, wüsste man nicht, ob eine eventuelle Differenz im Ausmaß der Hilfsbereitschaft auf die bittende Person oder auf die Sprache zurückzuführen ist. In diesem Experiment hat aber die gleiche Person die Bitte einmal in Schweizerdeutsch, das andere Mal in Hochdeutsch vorgetragen (Versuchsleitereffekte können auch bei diesem Design natürlich weiterhin bestehen). Es handelte sich um einen gebürtigen Deutschen, der in der Schweiz aufgewachsen ist und akzentfrei Hochdeutsch und Schweizer Dialekt spricht. Insgesamt wurden 109 Versuche durchgeführt, 55 auf Hochdeutsch, 54 auf Schweizerdeutsch. Das gesammelte Geld wurde an Greenpeace gespendet.

41\% der angesprochenen Personen kamen der Bitte nach, 59\% lehnten ab. Die Sprache hatte keinen nennenswerten Effekt auf das Ausmaß der Hilfeleistung. Schweizerdeutsch angesprochene Passantinnen und Passanten gaben in 41\% der Versuche zwei Franken, bei hochdeutsch Angesprochenen waren es 42\%. Auch wenn wir in der Auswertung nur die 80\% der Passantinnen und Passanten ohne ausländischen Akzent berücksichtigen, lässt sich keine signifikante Differenz feststellen. Sie beträgt dann zwar zwei Prozentpunkte zu Ungunsten der hochdeutschen Bittstellung, der geringe Unterschied bleibt aber klar nicht-signifikant ${ }^{6}$. Der Anteil „Spenden“ unter den Hochdeutsch bzw. Schweizerdeutsch angesprochenen Passanten ist aus Abbildung 2 ersichtlich, wobei nur Personen ohne ausländischen Akzent berücksichtigt wurden.

6 Hier und im Folgenden gehen wir von einem $\alpha$-Fehler von 0,05 aus. 
Abbildung 2: Ergebnisse der fünf Feldexperimente

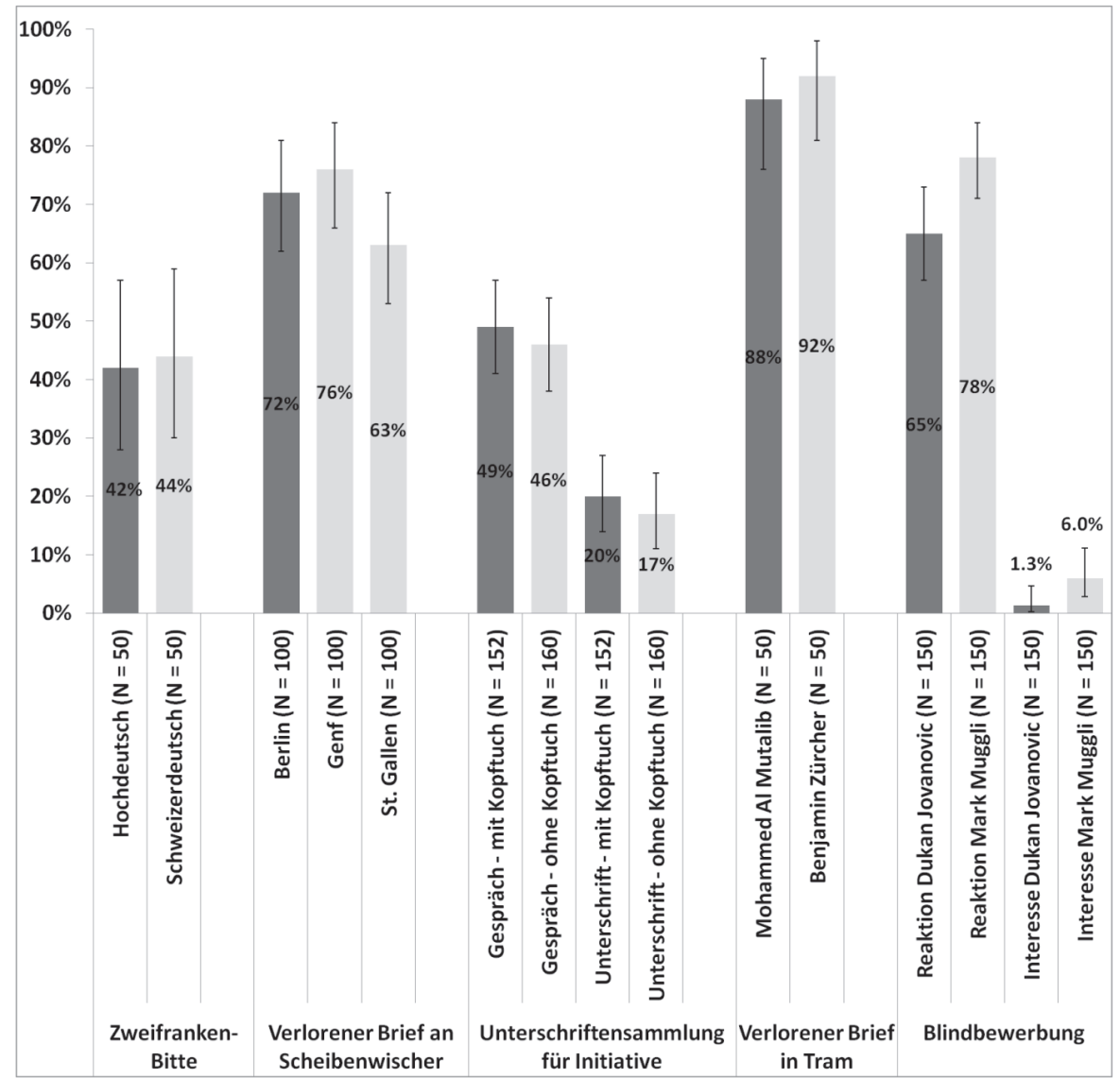

Anmerkung: Die Differenzen der Anteilswerte für die Experimente 1 bis 4 sind für $\alpha=0,05$ nicht signifikant. Bei Experiment 1 wurden nur Passanten ohne ausländischen Akzent berücksichtigt. Fishers exakter Test ( $p$ : zweiseitig; $p^{*}$ : einseitig): Exp. 1: $p=1,00 ; p^{*}=0,50$. Exp. 2: $p=0,13$. Exp. 3: a) $p=$ 0,$65 ; p^{*}=0,33$. b) $p=0,56 ; p^{*}=0,31$. Exp. 4: $p=0,74 ; p^{*}=0,37$. Exp. $5:$ a) $p=0,02 ; p^{*}=0,01$. b) $p$ $=0,06 ; p^{*}=0,03$. Fehlerbalken: exakte 95\%-Konfidenzintervalle aufgrund der Bionomialverteilung (Clopper-Pearson-Konfidenzintervalle).

\section{Experiment 2: Verlorene Briefe hinter dem Scheibenwischer ${ }^{7}$}

Die „Lost-Letter-Technik“ ist ein bekanntes, ursprünglich von Milgram, Mann und Harter (1965) eingeführtes nicht-reaktives Verfahren zur Einstellungsmessung. Briefe werden im öffentlichen Raum platziert (,,verloren“). Anhand von Differenzen in den Anteilen weitergeschickter Briefe (den „Rücklaufquoten“) kann auf die Sympathie gegenüber Merkmalen der Adresse geschlossen werden. Im Experiment von Milgram et al. waren die Briefe (scheinbar) gerichtet an die „Friends of Nazi Party“, „Friends of Communist Party“ und an das Rote Kreuz als Kontrollgruppe. Tatsächlich lagen die Anteile retournierter Briefe für die ersteren

7 Das Experiment wurde von Michael Jakob, Steven Meliopoulos, Michael Spuhler und Anh Minh Truong durchgeführt (siehe Jakob et al. 2010). 
beiden Gruppen mit nur 25\%, weit unter dem Rücklauf in der Kontrollgruppe. Der Finder eines Briefs ist weniger bereit, prosozial zu handeln und den verlorenen Brief in einen Postkasten zu stecken, wenn der Empfänger ihm unsympathisch erscheint. Natürlich bedeutet $25 \%$ Rücklauf bei den „Friends of Nazi Party“ nicht, dass jeder vierte Finder mit dieser (fiktiven) Gruppe sympathisieren würde. Selbst wenn dies keiner tut, könnten einige Finder der generellen Norm gefolgt sein, dass man einen verlorenen Brief unabhängig von Merkmalen des Adressaten weiterleiten soll. Interpretierbar als Grad der Sympathie sind nicht die absoluten Anteile weitergeschickter Briefe, wohl aber die Differenzen der Rücklaufquoten zwischen den einzelnen Versuchsbedingungen.

Ein Problem der Methode ist, dass Briefe oft von Gruppen gefunden werden oder natürlich auch mehrfach von verschiedenen Einzelpersonen. Ein in einer Telefonzelle platzierter Brief bleibt mit hoher Wahrscheinlichkeit so lange liegen, bis ein prosozialer Finder diesen mitnimmt und zurücksendet. Es kommt zur Selbstselektion prosozialer Finder. Deswegen empfiehlt es sich, gewissermaßen Stichproben von Findern auszuwählen. Z.B. kann man einen Brief hinter den Scheibenwischer eines geparkten Autos mit einem Zettel klemmen, auf dem der Hinweis steht, dass dieser Brief neben dem Fahrzeug gefunden wurde und vermutlich von dem Autofahrer verloren wurde (Abbildung 3). Damit geht der Brief an einen Finder, der nun entscheiden muss, ob er diesen weiterleiten soll oder nicht.

Auf diese Weise wurden 300 Briefe in elf Kreisen Zürichs hinter die Scheibenwischer von Autos gesteckt, und zwar abwechselnd Briefe mit drei unterschiedlichen Adressen. Das Design sieht drei Versuchsbedingungen vor: Eine Adresse in der Deutschschweiz, eine in der Westschweiz und eine in Deutschland. Ausgewählt wurden die Städte St. Gallen, Genf und Berlin. Insgesamt wurden je 100 Briefe pro Adresse verteilt. Als Adressat wurde der gleiche Name in allen Versuchsbedingungen gewählt. Die Wahl fiel auf den fiktiven Pascal Meyer, der an allen drei Orten hätte heimisch sein können. An den Postkästen der drei Adressen wurde ein entsprechender Vermerk angebracht, so dass die Post den fiktiven Adressaten erreichen konnte. Aufgrund der Verteilungsregeln (abwechselnd Briefe aus den drei Bedingungen unter Scheibenwischer klemmen) wurden alle anderen Merkmale (Uhrzeit, Ort, Wetter etc.) weitgehend konstant gehalten. Der Brief war frankiert. Für den Fall, dass Briefe geöffnet werden, befand sich im Kuvert ein Schreiben mit einer Einladung eines (fiktiven) Schachvereins „Rochade“ an Pascal Meyer. Auf dem Briefumschlag befand sich ein ,post it“ Zettel mit dem Hinweis: „Ihr Brief? Lag neben Auto.“ Die Handschrift auf dem Zettel unterschied sich von der Handschrift, in der der Brief adressiert war. 
Abbildung 3: Verlorener Brief hinter Scheibenwischer
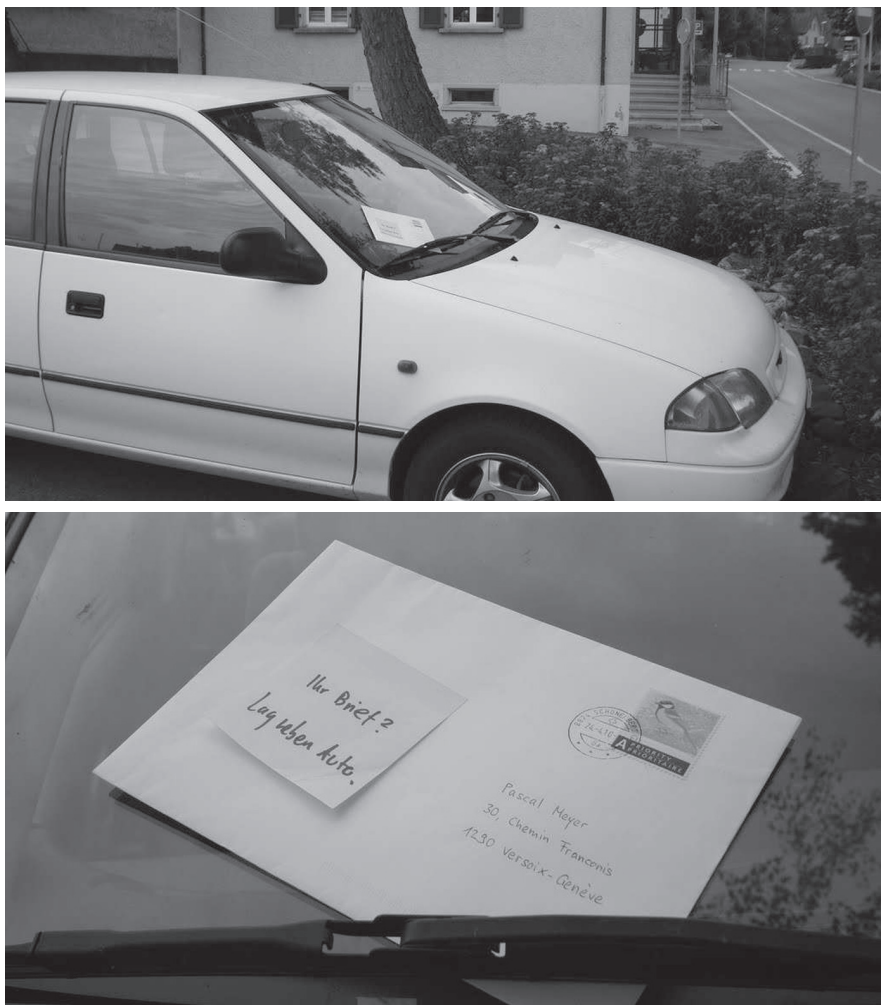

Insgesamt wurden 211 von 300 Briefen oder $70 \%$ weitergeschickt. 7 von 300 Briefen $(2,3 \%)$ wurden ersichtlich geöffnet. Von den je 100 Briefen pro Versuchsbedingung erreichten 63 St. Gallen, 76 Genf und 72 Berlin (Abbildung 2). Der Tendenz nach diskriminieren Zürcher eher ihre Landsleute im benachbarten St. Gallen. Doch muss hinzugefügt werden, dass die Differenzen nicht signifikant und womöglich Ergebnis zufälliger Schwankungen sind.

Beide Experimente ergeben keinen Anhaltspunkt für die Diskriminierung von Deutschen in der Schweiz. Wie sieht es aber mit anderen Ausländergruppen aus, insbesondere wenn Religion und ethnische Fremdheit das Potential für Vorurteile vergrößern könnten? Wir gehen dieser Frage mit den folgenden drei Experimenten nach.

\section{Experiment 3: Unterschriftensammlung für Initiative mit Kopftuch ${ }^{8}$}

In diesem Experiment bat in der Versuchsbedingung eine Studentin mit einem Kopftuch um eine Unterstützung bei einer politischen Aktion. Das Kopftuch wurde deutlich erkennbar in muslimischer Tradition gebunden (Abbildung 4). In der Kontrollbedingung sprach eine zweite Studentin ohne Kopftuch Passanten an. Die beiden Studentinnen traten gleichzeitig auf und wechselten die Rollen am zweiten Versuchstag. Sie warben für die Lohn-Initiative

8 Der Bericht basiert auf der Studie von Markus Glatt, Sabrina Maurer, Mischa Stalder, Schlomo Stokvis und Isabelle Straub (siehe Glatt et al. 2010). 
der Jungsozialisten („,1:12-Initiative“) an belebten Orten in Zürich. ${ }^{9}$ Registriert wurde, ob eine Person die Anfrage ignorierte oder aus Zeitgründen ablehnte oder ob sie sich auf ein Gespräch einließ. Weiterhin wurde festgehalten, ob sie die Initiative unterschrieben hatte. Gewiss hängt die Unterschrift auch von der politischen Einstellung ab. Wenn sich allerdings zeigen sollte, dass sich Personen in der Kontrollgruppe eher als in der Versuchsgruppe zu einem Gespräch bewegen lassen oder sogar unterschreiben, würde das Ergebnis für die Diskriminierungshypothese sprechen.

Abbildung 4: Kopftuchexperiment
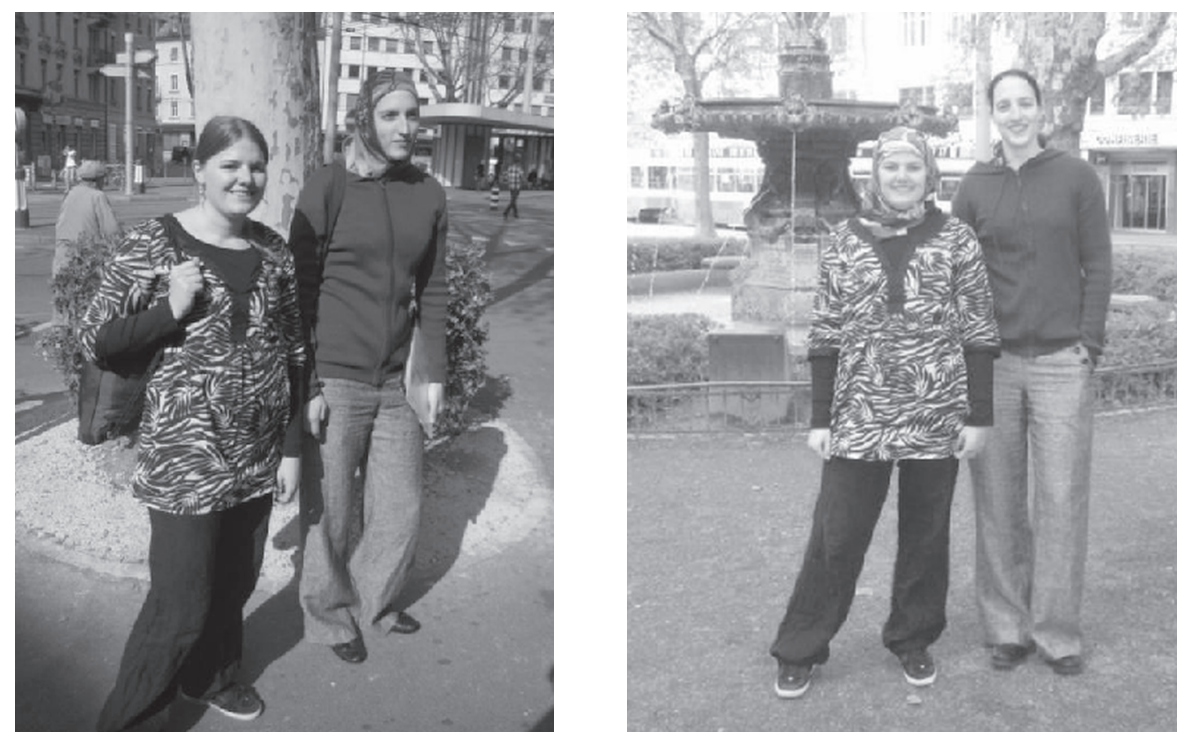

312 Personen wurden an den beiden Versuchstagen angesprochen. Von den 160 Personen der Kontrollgruppe ließen sich 73 (46\%) auf ein Gespräch ein. In der Versuchsgruppe wurden 152 Personen angesprochen. Von diesen nahmen 74 (49\%) das Gesprächsangebot an (Abbildung 2). Es unterschrieben 27 Personen in der Kontrollgruppe (17\% der Passanten, die den Kontakt akzeptiert hatten), in der Versuchsgruppe waren es 30 Personen (20\%). Die Unterschiede sind nicht signifikant. Ein Einfluss des Kopftuchs auf die Bereitschaft, ein Gespräch zu führen oder die Initiative zu unterschreiben, kann nicht nachgewiesen werden.

\section{Experiment 4: Verlorene Briefe im Tram ${ }^{10}$}

Mit der „Lost-Letter-Technik“ (siehe Experiment 2) werden in diesem Experiment die Einstellungen gegenüber arabischen Migranten erhoben. Im Unterschied zu Experiment 2 wurden die Briefe aber nicht hinter Scheibenwischern von Autos, sondern in Zürcher Trams deponiert. Der Nachteil der Methode ist, dass die Briefe nicht von bestimmten Zielpersonen

9 Mit der 1:12-Initiative wurde angestrebt, die Einkommensrelationen in Unternehmen zu verringern. Die Initiative forderte, das oberste Gehaltsniveau in einem Unternehmen auf maximal das 12 fache der untersten Lohngruppe zu begrenzen. Die Initiative wurde in der Abstimmung vom 24. November 2013 abgelehnt.

10 Durchgeführt von Melanie Thomas, Michael Vogel, Michael Waldner, Robert Weibel und Samy Ben Said (siehe Thomas et al. 2010). 
aufgefunden wurden. Andererseits betrifft dieses Problem sowohl die Kontrollgruppe als auch die Versuchsgruppe, so dass zwar nicht die absoluten Werte, wohl aber eventuell beobachtbare Differenzen Hinweise auf die Diskriminierungshypothese liefern können.

Es wurden 100 Briefe verteilt. 50 Briefe waren an den (fiktiven) Benjamin Zürcher (Kontrollgruppe), 50 Briefe an den (fiktiven) Mohammed Al-Muttalib adressiert. Je fünf Briefe mit dem schweizerischen und fünf Briefe mit dem arabischen Namen wurden in den Trams von 10 Zürcher Linien platziert. 46 Briefe (92\%) wurden an Benjamin Zürcher und 44 Briefe (88\%) an Mohammed Al-Mutalib weitergeleitet (Abbildung 2). Der Unterschied ist nicht signifikant.

\section{Experiment 5: Initiativbewerbungen bei Unternehmen ${ }^{11}$}

Mit dem Experiment wird der Frage nachgegangen, ob ein Bewerber aus Ex-Jugoslawien im Vergleich zu einem schweizerischen Bewerber auf einen Arbeitsplatz benachteiligt wird. Bei typischen Bewerbungsexperimenten werden Paare von Bewerbungen auf Stellenanzeigen hin an einen Arbeitgeber geschickt (,correspondence tests"). Dabei handelt es sich um zwei (fast) gleiche Beschreibungen (Lebensläufe, Qualifikation etc.), die sich nur im kausal interessierenden Merkmal wie z.B. Geschlecht, Nationalität usw. unterscheiden. Solche Feldexperimente wurden vielfach durchgeführt; ein Überblick findet sich bei Riach und Rich (2002). Wir haben mit 300 Initiativbewerbungen an Unternehmen aus dem Finanz- und Industriesektor einen etwas anderen Weg gewählt. Initiativbewerbungen sind u.E. weniger heikel als Scheinbewerbungen auf Stellenanzeigen, da eine „experimentelle“ Bewerbung (und eine Absage des Bewerbers im Falle einer Einladung) durchaus die tatsächlichen Chancen von Kandidatinnen und Kandidaten für eine Stelle beeinflussen kann. Es ist forschungsethisch sicher nicht unproblematisch, wenn ein Experiment einen Einfluss auf das Resultat eines Auswahlverfahrens hat (Diekmann 2007). Bei Initiativbewerbungen ist dies eher unwahrscheinlich. Weiterhin ist zu bemerken, dass die Feldexperimente, gleich ob Experimente mit Paaren von Bewerbungen auf Stellenanzeigen oder Initiativbewerbungen, zwar Hinweise auf das Ausmaß von Diskriminierung, nicht aber auf deren genaue Ursachen wie z.B. Ungleichbehandlung durch „statistische Diskriminierung“ geben können (List 2006).

Die Bewerbungen wurden an Deutschschweizer Unternehmen mit mindestens 50 Angestellten gesandt. Die Unternehmen wurden zufällig aus dem Betriebs- und Unternehmensregister (BUR) des Bundesamts für Statistik gezogen. Dabei wurde die Stichprobe nach Industrie- und Finanzsektor geschichtet. An jedes der Unternehmen in der Stichprobe wurde sodann eine Bewerbung geschickt.

Bei 150 Bewerbungen hieß der Kandidat Mark Muggli, bei 150 weiteren Bewerbungen Dukan Jovanović. Eine Bewerbung bestand aus einem Begleitschreiben und einem (fiktiven) Lebenslauf (Abbildung 5). Das Begleitschreiben wurde in mehreren Varianten formuliert und von Experten geprüft. Die beiden Namen wurden gleichmäßig auf die Varianten des Begleitschreibens verteilt. Gemäß dem Lebenslauf hatte der Bewerber eine kaufmännische Lehre abgeschlossen (,KV-Absolvent") und kürzlich eine Rekrutenschule besucht. Der Lebenslauf enthielt Angaben zur Nationalität, Ausbildung, Berufserfahrung, zum Alter, der Schulkarriere und zu Referenzen sowie ein in allen Bewerbungen gleiches Foto. Für beide Bewerber wurde die Schweizer Nationalität angegeben. Wer die Unterlagen studierte, musste den Eindruck gewinnen, dass Mark Muggli ein Schweizer ohne und Dukan Jovanović ein Schweizer mit Migrationshintergrund ist. Alle Bewerbungen wurden zufällig je einem Unternehmen zugewiesen.

11 Die Studie haben Nora Reinhardt, Silvia Richina und Alena Schmidt unternommen (siehe Reinhardt et al. 2010). 
Abbildung 5: Beispiel einer Initiativbewerbung

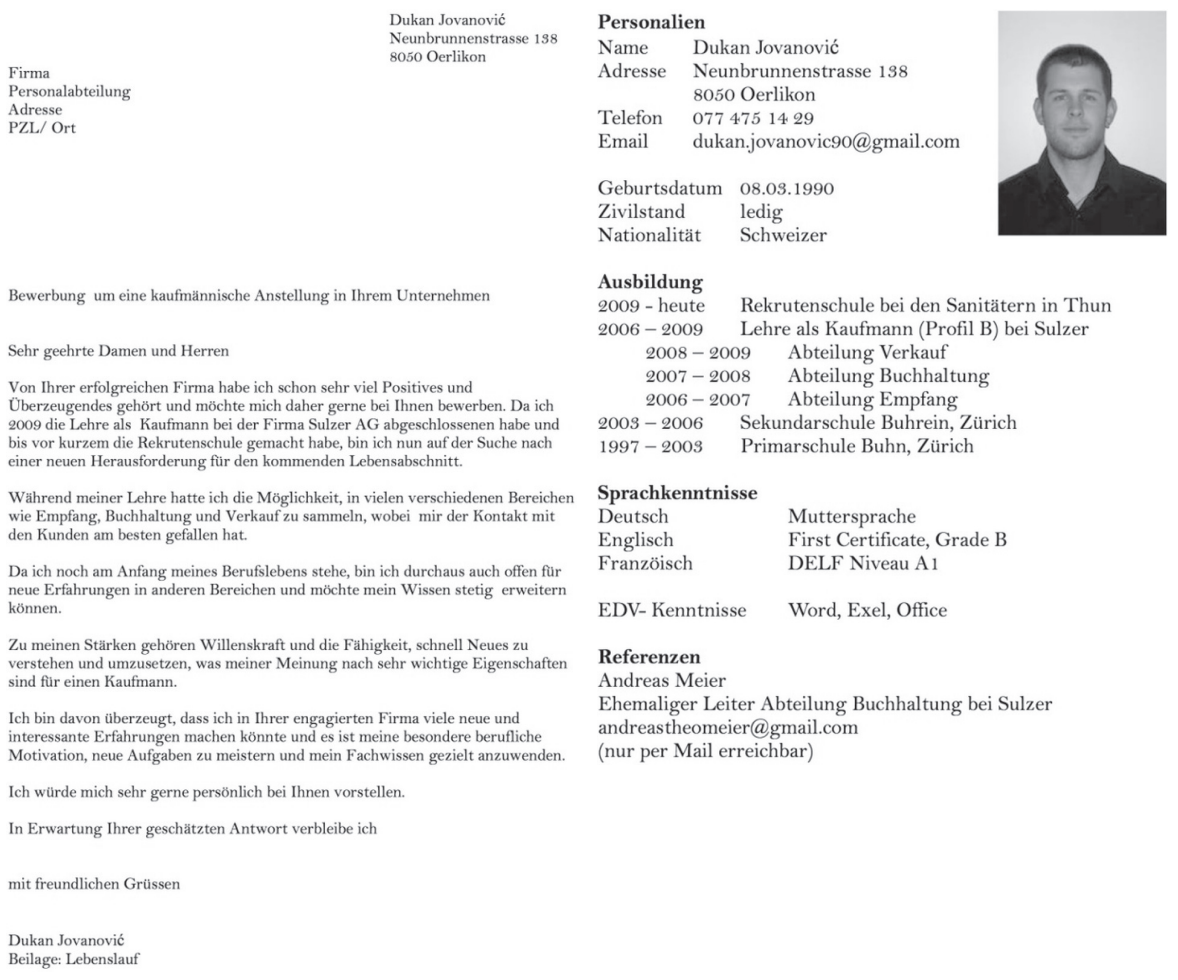

Die Erfolgschancen von Initiativbewerbungen sind naturgemäß relativ gering. Auf $85 \mathrm{Be}-$ werbungen erfolgte keine Antwort, bei 204 Bewerbungen war die Antwort negativ. Bei den restlichen elf Bewerbungen wurde um weitere Unterlagen gebeten (5) oder eine Prüfung der Unterlagen (1) bzw. die Weiterleitung (2) in Aussicht gestellt. Drei Bewerbern wurde ein Stellenangebot unterbreitet. Eine Reaktion (die aber auch negativ sein konnte) erfolgte bei Mark Muggli bei 78\% und bei Dukan Jovanović bei $65 \%$ der Bewerbungen, was einem statistisch signifikanten Unterschied entspricht ( $p=0,01$; einseitiger Test).

Wie verteilen sich nun die elf Bewerbungen, bei denen Interesse signalisiert wurde, auf die beiden Namen? Hier zeigt sich eine Bevorzugung von Mark Muggli gegenüber Dukan Jovanović. Neun Mal besteht Interesse an der Bewerbung von Mark Muggli, nur zwei Mal besteht Interesse an der Bewerbung von Dukan Jovanović (Abbildung 2).

Die Differenz von 6 versus 1,33 Prozent (bei je 150 Beobachtungen) ist für eine Irrtumswahrscheinlichkeit von $\alpha=0,05$ signifikant ( $p=0,03$; einseitiger Test). Allerdings ist die Verteilung extrem schief und die absolute Zahl interessierter Reaktionen sehr gering. Das Feldexperiment liefert nur einen Hinweis auf Diskriminierung. Um Aussagen mit größerer Gewissheit machen zu können, sind bei Initiativbewerbungen wesentlich größere Fallzahlen erforderlich.

Wie bereits erwähnt sind Bewerbungsexperimente anhand einer Stichprobe von Stellenanzeigen eine Alternative. Für solche Experimente genügen geringere Fallzahlen. Fibbi, Lerch und Wanner (2006) haben anstelle von Initiativbewerbungen ein Experiment mit Bewerbun- 
gen auf Zeitungsannoncen unternommen. Hierbei wurden jeweils Paare von (fast gleichen) Lebensläufen an das annoncierende Unternehmen geschickt, wobei jeweils der Name und die Herkunft variiert wurden. Bei allen (fiktiven) Bewerbungen handelte es sich um junge Männer, die den Abschluss einer Lehre vorzuweisen und ihre gesamte schulische Laufbahn in der Schweiz absolviert hatten. Die Bewerber ausländischer Herkunft waren jedoch nicht in der Schweiz geboren. Es zeigte sich eine massive Diskriminierung der türkischen und, stärker noch, der jugoslawischen Herkunft. Die Diskriminierung von Bewerbern jugoslawischer Herkunft war erheblich in der französischen Schweiz, aber noch wesentlich stärker in der Deutschschweiz. Die Diskriminierung junger Männer türkischer Herkunft in der Schweiz lag zudem über der Diskriminierungsrate, die in einem ähnlichen Experiment in Deutschland ermittelt wurde. Für Bewerber aus portugiesischen Familien war die Diskriminierung in dem Experiment von Fibbi et al. dagegen relativ gering und nicht signifikant. Diese Studie macht deutlich, dass auf dem Arbeitsmarkt in der Schweiz substanzielle Diskriminierung zumindest gegenüber Bewerbern besteht, deren Familien aus der Türkei oder Jugoslawien in die Schweiz eingewandert sind.

\section{Diskussion}

Vier von fünf Feldexperimenten ergeben keinen Hinweis auf die Diskriminierung von Ausländerinnen und Ausländern in der Schweiz im alltäglichen Umgang miteinander. Insbesondere konnte keine Diskriminierung von Deutschen im Vergleich zu Inländern festgestellt werden. Dass wir keine Diskriminierung im Alltag feststellen konnten, spricht immerhin für ein gewisses Ausmaß der Toleranz in der größten Stadt der Schweiz, die einen erheblichen Zustrom von Ausländern erlebt. Ob ein Kopftuch getragen wurde oder nicht, ob Schweizer oder deutsche Adresse, Hochdeutsch oder Dialekt; in all diesen Fällen war kein systematischer Einfluss auf das Ausmaß kleiner Hilfeleistungen zu beobachten. Ob Gleiches auch auf dem Lande gilt oder - bezüglich des Kopftuchexperiments - in ausländischen Metropolen wie Paris oder Berlin, wäre wissenswert. Zu bedenken ist schließlich, dass die Abstimmung über die Masseneinwanderungsinitiative vom Februar 2014 in der Stadt Zürich relativ wenige Anhänger hatte. In der Stadt stimmten 33,4\% für die Initiative, im Kanton Zürich 47,3\%. In der Innerschweiz lagen dagegen die Zustimmungswerte bei etwa 60\%, im Tessin waren es sogar $68,2 \%$. Es wäre also gut möglich, dass Nicht-Diskriminierung im relativ liberalen Zürich die Regel ist, während es sich auf dem Lande und insbesondere in der Innerschweiz anders verhält. Diese und weitere Hypothesen könnten durch Feldexperimente untersucht werden. Dabei wäre durchaus Raum für methodische Erweiterungen, etwa die Kombination von Feldexperimenten mit einer Befragung. ${ }^{12}$

Ein weiteres Bewerbungsexperiment liefert dagegen einen Anhaltspunkt, dass Bewerber aus Ex-Jugoslawien trotz Schweizer Nationalität gegenüber Bewerbern ohne Migrationshintergrund auf dem Arbeitsmarkt benachteiligt sein könnten. Einen deutlichen Beleg für die Arbeitsmarktdiskriminierung türkischstämmiger und ex-jugoslawischer Migranten liefert zudem die Studie von Fibbi, Lerch und Wanner (2006). Interessant wäre dabei zu wissen, welche weiteren Ausländergruppen auf dem Arbeitsmarkt benachteiligt sind. Gehören Deutsche dazu oder werden diese im Gegenteil sogar bevorzugt? Gibt es Interaktionseffekte mit der Qualifikation der sich bewerbenden Person oder mit dem Berufsfeld der zu besetzenden Stelle? Bewerbungsexperimente könnten zu diesen Fragen Auskunft geben. Wir bevorzugen allerdings die Methodik von Initiativbewerbungen, da Bewerbungen auf Annoncen in das soziale Geschehen stärker eingreifen und das Ergebnis einer Auswahl durch das Unternehmen durchaus beeinflussen können.

12 Diesen Hinweis verdanken wir einem anonymen Gutachten. Siehe zur Kombination von Feldexperimenten mit anderen Methoden auch Diekmann (2007: Kap. XIII). 
Arbeitsmarktdiskriminierung ist in der Regel folgenreicher als die Verweigerung einer Bitte im Alltag, obwohl auch bei letzterem Verhalten systematische Benachteiligung, wenn sie bestünde, das soziale Klima in einer Gesellschaft vergiften kann. Eine mögliche Erklärung wäre, dass bei Low-Cost-Aktivitäten wie einer wenig aufwändigen prosozialen Handlung (ein Gespräch führen, eine Unterschrift bei einer politischen Initiative leisten, zwei Franken geben, einen Brief weiterleiten) egalitär gehandelt wird, bei Entscheidungen mit größeren Kostenfolgen dagegen sehr wohl diskriminiert wird. Bei einer einmaligen Interaktion kann man allfällige Vorurteile schon mal zurückstellen. Der Konflikt zwischen der Norm zur Hilfeleistung und dem Vorurteil wird dann zu Gunsten der Normbefolgung gelöst. Dauerhafte Interaktionen zum Beispiel im Rahmen eines Arbeitsverhältnisses sind dagegen für Personen mit Vorurteilen (als auch für Personen, auf die sich die Vorurteile beziehen) mit höheren Kosten verbunden. In diesem Fall kann das Vorurteil die Oberhand gewinnen. Im Gegenzug entstehen für diskriminierende Arbeitgeber jedoch ökonomische Nachteile. Der Preis des Vorurteils ist, wie der Ökonom Gary Becker herausgefunden hat, dass öfter gut qualifizierte Bewerber zugunsten weniger gut qualifizierter Bewerber abgewiesen werden. Dadurch profitieren auf Wettbewerbsmärkten vorurteilsfreie Unternehmen, während diskriminierende Unternehmen Gewinneinbußen tragen müssen (Becker 1957). Auch die Vermietung einer Wohnung ist folgenreich sowohl für Vermieter als auch Wohnungssuchende. Denn auch bei der Akzeptanz von Mietern ist zu vermuten, dass Diskriminierung wegen der Dauerhaftigkeit von Interaktionen eine größere Rolle spielen wird als bei einmaligen Begegnungen in Alltagssituationen. Die theoretische Erklärung von Vorurteilen hebt auf die fünf Faktoren Zugehörigkeit, Verstehen, Kontrolle, Selbstwertgefühl und Vertrauen ab (Fiske 2000). Größeres Vertrauen wird den Mitgliedern der eigenen Gruppe im Unterschied zur Fremdgruppe entgegengebracht. Diese Vertrauensdifferenz kann Diskriminierung verstärken. Gerade also bei sozialen Interaktionen, bei denen ein Vertrauensproblem auftritt wie z.B. bei der Vermietung einer Wohnung oder bei der Vergabe von Krediten, wäre demnach das Auftreten von Mechanismen der Diskriminierung zu erwarten. $\mathrm{Zu}$ vermuten ist, dass deshalb Vorurteile in solchen Situationen in stärkerem Maße zu beobachten sein werden als in einfachen Alltagssituationen - eine Hypothese, der gleichfalls in Feldexperimenten nachgegangen werden könnte.

\section{Literatur}

Becker, G.S. (1957): The Economics of Discrimination, Chicago / IL.

Beglinger, M. (2013): Alle wollen hierher. Das Magazin des Tagesanzeigers Zürich Nr. 36, 13. 9. 2013, S. 24-33.

Bungard, W. / H.E. Lück (1974): Forschungsartefakte und nicht-reaktive Meßverfahren, Stuttgart.

Diekmann, A. (2007): Empirische Sozialforschung. Grundlagen, Methoden, Anwendungen, Reinbek.

Fibbi, R. / M. Lerch / P. Wanner (2006): Unemployment and Discrimination against Youth of Immigrant Origin in Switzerland: When the Name Makes the Difference, in: Journal of International Migration and Integration 7, S. 351-366.

Fiske, S.T. (2000): Stereotyping, Prejudice, and Discrimination at the Seam Between the Centuries: Evolution, Culture, Mind, and Brain, in: European Journal of Social Psychology 30, S. 299-320.

Glatt, M. / S. Maurer / M. Stalder / S. Stokvis / I. Straub (2010): Mit dem Kopftuch unterwegs. Werden kopftuchtragende Personen im Alltag der Stadt Zürich diskriminiert?, Studentischer Forschungsbericht, betreut durch B. Jann und M. Naef, ETH Zürich.

Helbling, M. (2011): Why Swiss Germans Dislike Germans. Opposition to Culturally Similar and Highly Skilled Immigrants, in: European Societies 13, S. 5-27.

Hewstone, M. (2003): Intergroup Contact. Panacea for Prejudice?, in: The Psychologist 16, S. 352-355. 
Jakob, M. / S. Meliopoulos / M. Spuhler / A.M. Truong (2010): Wie deutschfeindlich ist Zürich? Spurensuche mittels verlorenen Briefen, MTU-Arbeit, betreut durch A. Diekmann, ETH Zürich.

Jann, B. (2014): Fünf Feldexperimente zu ethnischer Diskriminierung. Codebuch und Dokumentation, Institut für Soziologie der Universität Bern.

Jetten, J. / R. Spears / A.S.R. Manstead (1998): Defining Dimensions of Distinctiveness. Group Variability Makes a Difference to Differentiation, in: Journal of Personality and Social Psychology 74, S. 1481-1492.

Krumpal, I. (2012): Estimating the Prevalence of Xenophobia and Anti-Semitism in Germany: A Comparison of Randomized Response and Direct Questioning, in: Social Science Research 41, S. 1387-1403.

List, J.A. (2006): Field Experiments: A Bridge Between Lab and Naturally Occurring Data. Advances in Econometric Analysis and Policy 6.

Milgram, S. / L. Mann / S. Harter (1965): The Lost-Letter-Technique: A Tool of Social Research, in: Public Opinion Quarterly 29, S. 437-438.

Pettigrew, T.F. / L.R. Tropp (2008): How Does Intergroup Contact Reduce Prejudice? Meta-Analytic Tests of Three Mediators, in: European Journal of Social Psychology 38, S. 922-934.

Riach, P.A. / J. Rich (2002): Field Experiments of Discrimination in the Market Place, in: The Economic Journal 112, S. 480-518.

Reinhardt, N. / S. Richina / A. Schmidt (2010): Diskriminierung im Arbeitsmarkt: Bewerbungsexperimente. Studentischer Forschungsbericht, betreut durch B. Jann und M. Näf, ETH Zürich.

Stolz, J. (2001): Einstellungen zu Ausländern und Ausländerinnen 1969 und 1995: Eine Replikationsstudie, in: H.-J. Hoffmann-Nowotny (Hrsg.), Das Fremde in der Schweiz, Zürich.

Tajfel, H. (1981): Human Groups and Social Categories, Cambridge.

Tajfel, H. / M.G. Billig / R.P. Bundy / C. Flament (1971): Social Categorization and Intergroup Behavior, in: European Journal of Social Psychology 1, S. 149-178.

Thomas, M. / M. Vogel / M. Waldner / R. Weibel / S.B. Said (2010): Lost-Letter-Experiment. Werden Menschen mit arabischem Namen diskriminiert? Studentischer Forschungsbericht, betreut durch B. Jann und M. Näf, ETH Zürich.

Webb, E.J. / D.T. Campbell / R.D. Schwartz / L. Sechrest (1966): Unobtrusive Measures. Nonreactive Research in the Social Sciences, London.

Zimdars, C. / O. van der Reijden / F. Hoppeler / M. Bieli / L. Weber (2010): Deutschenfeindlichkeit in Zürich. Studentischer Forschungsbericht, betreut durch B. Jann und M. Näf, ETH Zürich.

Prof. Dr. Andreas Diekmann Matthias Näf Eidgenössische Technische Hochschule Professur für Soziologie Clausiusstr. 50

8092 Zürich, Schweiz diekmann@soz.gess.ethz.ch

Prof. Dr. Ben Jann

Universität Bern Institut für Soziologie

Fabrikstr. 8

3012 Bern

jann@soz.unibe.ch 